\title{
The Patent fact about Alcohol and Getting Stroke: A Case Control Study
}

\section{Kuntalkumar H Patel ${ }^{1}$, Shreyashkumar Gandhi ${ }^{2}$, Dhruti Vaidya ${ }^{3}$}

${ }^{1}$ Assistant Professor, ${ }^{2}$ Associate Professor, Department of Preventive and Social Medicine

${ }^{3}$ Assistant Professor, Department of Pharmacology,

Dr. Kiran C Patel Medical College and Research Institute, Bharuch, Gujarat, India.

Correspondence : Dr. Shreyashkumar Gandhi, Email: drshreyash77gandhi@gmail.com

\begin{abstract}
:
Introduction: Globally 17.9 million people die each year from CVDs (Cardio Vascular Diseases) and $85 \%$ of all CVD deaths are due to heart attacks and strokes. According to the World Health Organization (WHO), 15 million people worldwide suffer from stroke every year and after ischemic heart disease, stroke is the second most leading cause of death. Objective: To study alcohol consumption as a risk factor in patients with acute strokes. Method: Case control study was conducted among 148 newly diagnosed cases of CT scan / MRI-confirmed stroke and 148 non-stroke controls attending the tertiary hospital of central Gujarat. Sample size calculation was done, assuming the power ratio of the number of controls same as numbers of cases i.e.,1:1, specifying values for two-sided confidence level=95\% (error=5\%), power of study $=80 \%$, odd ratio $=2.15$, percent of cases with exposure $=82 \%$ and percent of controls with exposure $=68 \%$. Patients who are willing to participate in the study were included. The data were analyzed using the EPI INFO TM 7.0.8.0 software. Results: Out of the total 148 cases, 100 ( 67.56 per cent) were male and 48 (32.43 per cent) were female. The odds of getting stroke among alcoholic was two times more as compared to non-alcoholic patients. Heavy alcohol drinkers have a significant association. Conclusion: According to the study results male married patients who drank heavily had a higher risk of stroke than light/moderate alcoholics.
\end{abstract}

Key words: Alcohol, Cerebro vascular accident, Stroke

\section{Introduction:}

A stroke is a non-communicable disease of increasing importance. According to the World Health Organization (WHO), 15 million people worldwide suffer from a stroke every year. Globally, 6.5 million stroke deaths occurred in 2013, making National Programme for Prevention and Control of Cancer, Diabetes, Cardiovascular Diseases and Stroke (NPCDCS) guideline ${ }^{[2]}$, "Stroke or Cerebrovascular accident means that part of the brain is suddenly damaged. If any artery in the brain becomes blocked by a thrombus, it causes a stroke or an artery in the brain

\begin{tabular}{|c|l|}
\hline Quick Response Code & Access this article online \\
\hline 口ifitis: & $\begin{array}{l}\text { Website : } \\
\text { www.healthlinejournal.org }\end{array}$ \\
\cline { 2 - 2 } & DOI : \\
10.51957/Healthline_219_2021
\end{tabular}

leaks, then too it damages the brain and causes a stroke".

Stroke is a medical emergency that can cause permanent neurological harm and death. There are two kinds of stroke episodes depending on the location of the stroke in the brain. A minor stroke episode Transiant Ischaemic Attack (TIA) may cause minor issues, such as weakness in the arm or leg. A significant episode of the stroke can lead to paralysis or even death. Many stroke patients are left with weakness on one side of the body, speech difficulties, incontinence and/or bladder problems.

\section{How to cite this article :}

Patel K, Gandhi S, Vaidya D. The patent fact about alcohol and getting stroke: A Case Control Study. Healthline. 2021; 12(2):28-32. 
There are two significant stroke subtypes (i) ischemic stroke (ii) hemorrhagic stroke. Ischemic stroke happens as a consequence of obstruction within a blood vessel that supplies blood to the brain, which may be thrombotic or embolic. It accounts for $87 \%$ of all stroke cases. ${ }^{[3]}$ Stroke is a multi-factorial disease that happens owing to a mixture of different risk variables, all of which may not necessarily be present at the moment of its incidence.

According to the NPCDCS guideline ${ }^{[2]}$, behavioral risk factors include unsanitary diet, physical inactivity, heavy alcohol consumption, cigarette addiction and stress, and physiological risk factors include enhanced Body Mass Index (BMI), hypertension, high blood sugar and hypercholesterolemia, which are modifiable risk factors. The non-modifiable risk variables include age, gender and family genetics. Previous studies recognized alcohol is one of the independent risk factors that are extensively associated with stroke. ${ }^{[4-7]}$ Research shows that drinking large amounts of alcohol will substantially increase your risk of stroke, since alcohol leads to a number of medical conditions that are risk factors for stroke. Very few studies have been conducted in developing nations such as India related to effect of alcohol and stroke. Present study was conducted with objective to study alcohol consumption as a risk factor in patients with acute strokes.

\section{Method:}

A case control study was performed in the Medicine and Medical Intensive Care Unit (MICU) departments from January 2018 to June 2018 in one of the tertiary care hospital of central Gujarat. Cases were those whose diagnosis of a stroke had been confirmed by CT Scan / MRI. The recruits of cases in the study were incident cases (first ever in the lifetime) within 72 hours after admission. Each and every confirmed stroke case patient who arrived during the study period and agreed to participate in the study were enrolled. Patients who were admitted to the same ward for circumstances other than stroke were chosen for the incorporation of control. One control was chosen for each stroke case. For feasibility of study controls were chosen from the patients admitted to the Department of Medicine / Ward, without a stroke such as Typhoid, Hepatitis (Jaundice), Diarrhoea, Chronic Obstructive Pulmonary Discase (COPD), Malaria, etc. Cases and controls patient not ready to participate in the research and patients with transient ischaemic attack, poisoning, trauma-related symptoms and past history of IHD were excluded from the study.

Medcalc software was used to calculate sample size. Assuming the power ratio of the number of controls same as numbers of cases specifying values for two-sided confidence level 95\% and odd ratio 2.15 which was the minimum risk factor in previous study (Obesity, odds ratio-2.15) ${ }^{[8]}$ According to the reference study, obesity has the lowest odds ratio of all risk factors for stroke, so we chose it for sample size calculation in the current study. So, considering 82 percent of cases with exposure and 68 percent of controls with exposure, the total sample size was 296 including 148 cases and 148 controls have been selected. Matching was done for the gender. Confounding factors include age, smoking, obesity, and other risk factors.

The interviewer was fully informed about the study and its purpose, the risks or benefits of participation and confidentiality of the information. If the patient was unable to communicate sufficiently to complete the study questionnaire, proxy respondent was used. The valid proxy respondent was a spouse (husband/wife) or first-degree relative (a person's parent, sibling, relative or more than 18 years old son/daughter) who was living in the same home or was aware of the patient's previous medical history and present treatments.

This study was reviewed and approved by the Institutional Ethics Committee for Human Research (IECHR), prior to the initial interview, written consent was obtained from the participants.

Physicians operationally defined "light" drinking as 1 drinks/day, "moderate" drinking as 2 drinks/day, and "heavy" drinking as $>3$ drinks / day. ${ }^{[9]}$

\section{Results:}

The present study was conducted to study alcohol 
consumption as a risk factor in patients with acute strokes admitted in the Medicine wards and Medical Intensive Care Unit (MICU) from January 2018 to June 2018 of one of the teaching hospitals of Vadodara.

As shown in Table 1, total 148 cases of Stroke and 148 controls were analysed. Among the cases $67.56 \%$ were males and $32.43 \%$ were females. There were 135 (91.22\%) cases and 127 (85.81\%) controls who were Hindus followed by $13(8.78 \%)$ cases and 21 (14.19\%) controls who were Muslim by religion. Out of 296 study subjects, 120 (81.08\%) cases were married, 4 (2.70\%) were unmarried while 142 (95.95\%) controls were married and two (1.35\%) were unmarried.

Table 1: Socio-Demographic Characteristics of cases and controls

\begin{tabular}{|c|c|c|}
\hline Variables & Cases (n=148) & Controls (n=148) \\
\hline Gender distribution \\
\hline Male & $100(67.57 \%)$ & $100(67.57 \%)$ \\
\hline Female & $48(32.43 \%)$ & $48(32.43 \%)$ \\
\hline Religion of Patients \\
\hline Hindu & $135(91.22 \%)$ & $127(85.81 \%)$ \\
\hline Muslim & $13(8.78 \%)$ & $21(14.19 \%)$ \\
\hline Marital Status \\
\hline Married & $120(81.08 \%)$ & $142(95.95 \%)$ \\
\hline Unmarried & $4(2.70 \%)$ & $2(1.35 \%)$ \\
\hline Other* & $24(16.22 \%)$ & $4(2.70 \%)$ \\
\hline
\end{tabular}

* Other (Widow, Widower and Divorce)

As shown in Table 2, 51 (34.46\%) of the cases and $32(21.62 \%)$ of the controls consumed alcohol. The crude odds ratio was 1.91, that is odds (chance) of getting stroke among alcoholic was two times more as compared to non-alcoholic patients.

Table 2: Distribution of study subjects according to history of alcohol consumption

\begin{tabular}{|c|c|c|}
\hline Alcohol consumption & Cases (n=148) & Controls (n=148) \\
\hline Yes & $51(34.46 \%)$ & $32(21.62 \%)$ \\
\hline No & $97(65.54 \%)$ & $116(78.38 \%)$ \\
\hline OR - $1.91,95 \%$ CI -1.14 to 3.20 P value -0.01 \\
\hline
\end{tabular}

Cases and controls in the study were distributed on the basis of alcohol consumption, whether daily or occasionally. Table 3 shows, four (8\%) cases and five (16\%) controls consumed alcohol daily. The crude odds ratio was 0.46 , that is odds (chance) of getting stroke was lower in occasional alcohol drinker as compared to daily alcohol drinker.

Table 3: Distribution of study subjects according to frequency of alcohol consumption

\begin{tabular}{|c|c|c|}
\hline Current use & Cases (n=51) & Controls (n=32) \\
\hline Daily* & $4(7.84 \%)$ & $5(15.63 \%)$ \\
\hline Occasionally** & $47(92.16 \%)$ & $27(84.37 \%)$ \\
\hline OR - 0.46, 95\% CI - 0.11 to 1.86, P value-0.28 \\
\hline
\end{tabular}

* Daily - Heavy drinker

** Occasionally - Light / Moderate drinker

As shows in Table 4, 4 (7.84\%) cases consumed heavy alcohol followed by 8 (15.69\%) who consumed moderate amount of alcohol and only 39 (76.47\%) case consumed light amount of alcohol. In controls 5 (15.63\%) consumed heavy amount of alcohol, followed by $10(31.26 \%)$ consumed moderate alcohol and 17 (53.13\%) consumed light amount of alcohol which was statistically significant.

Table 4: Distribution of study subjects according to severity of alcohol consumption

\begin{tabular}{|c|c|c|}
\hline $\begin{array}{c}\text { Alcohol } \\
\text { consumption }\end{array}$ & $\begin{array}{c}\text { Cases } \\
(\mathbf{n = 5 1 )}\end{array}$ & $\begin{array}{c}\text { Controls } \\
(\mathbf{n = 3 2})\end{array}$ \\
\hline Heavy drinker & $4(7.84 \%)$ & $5(15.63 \%)$ \\
\hline Moderate drinker & $8(15.69 \%)$ & $10(31.26 \%)$ \\
\hline Light drinker & $39(76.47 \%)$ & $17(53.13 \%)$ \\
\hline$\chi 2$ (trend) Value - 4.11, DF - 1, p- 0.04 \\
\hline
\end{tabular}

Figure 1: Distribution of study subjects according to duration of alcohol consumption

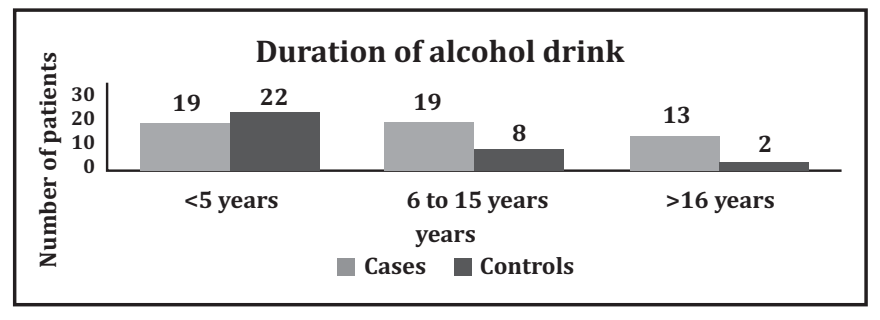

$\chi 2$ (trend) Value - 8.78, DF - 1, p - 0.003 
Cases and controls were further distributed based on the alcohol consumption period. (Figure1) Total 19 (37.25\%) cases had consumed alcohol for less than five years of duration followed by 19 (37.25\%) who consumed alcohol for 6 to 15 years of duration and only 13 (25.49\%) case consumed alcohol for $>16$ years of duration. In controls 22 (68.75\%) consumed alcohol for less than five years of duration, followed by eight (25\%) consumed alcohol for 6 to 15 years of duration and only two (6.25\%) consumed alcohol for $>16$ years of duration which was statistically significant.

\section{Discussion:}

The present study was conducted in the Medicine wards and MICU from January 2018 to June 2018 of SSG Hospital Vadodara, one of the tertiary care hospital of central Gujarat.

In the study, 51 (35\%) of the cases and 32 (22\%) of the controls were alcoholic. The crude odds ratio was 1.91, that is odds (chance) of getting stroke among alcoholic was virtually two times more in alcoholic as compared to non-alcoholic. This is statistically significant association. Similar result found in a meta-analytic dose-response study done by Zhang $C$ et al (2014) which included 27 prospective studies that reported data on $14,25,513$ people. Low alcohol intake was associated with a reduced risk of total stroke and stroke mortality, but it had no significant effect on haemorrhagic stroke. Moderate intake of alcohol had little or no effect on the risk of total stroke, haemorrhage stroke, ischemic stroke and mortality of strokes. Heavy intake of alcohol was associated with an increased risk of total stroke, but had no significant effect on haemorrhagic stroke, ischemic stroke, and stroke mortality. ${ }^{[10]}$

Other meta-analysis conducted by Larsson SC et al (2016) included 27 prospective studies with ischemic stroke (25 studies), intra cerebral haemorrhage (11 studies) and/or subarachnoid haemorrhage (11 studies). Light and moderate alcohol consumption was associated with a lower risk of ischemic stroke, where as high and heavy drinking was associated with an increased risk; the overall RRs were 0.90 (95\% CI, 0.85-0.95) for less than 1 drink/day, 0.92 (95\% CI, 0.87-0.97) for 1-2 drinks/day, 1.08 (95\% CI, 1.01-1.15) for more than 24 drinks/day, and 1.14 (95\% CI, 1.02-1.28) for more than 4 drinks/day. Light and moderate alcohol consumption was not related to any subtype of haemorrhagic stroke. High alcohol consumption ( $>2$ - 4 drinks / day) was associated with a non significant increased risk of both haemorrhagic stroke subtypes, and the relative risk of heavy drinking ( $>4$ drinks/day) for intracerebral haemorrhage was 1.67 (95\% CI, 1.25-2.23) and 1.82 (95\%CI, 1.18-2.82) for subarachnoid haemorrhage. ${ }^{[11]}$

In the present study, four (8\%) of the cases and five $(16 \%)$ of the controls consumed alcohol daily. The crude odds ratio was 0.46 , that is odds (chance) of getting stroke were lower in occasional alcohol drinker as compared to daily heavy alcohol drinker. This is statistically not significant association. In a study conducted by Lee SJ et al (2015), light to moderate alcohol intake of 3 to 4 beverages ( 1 drink = $10 \mathrm{~g}$ ethanol) per day was substantially associated with lower risk of ischaemic stroke after accounting for potential confounders. ${ }^{[12]}$

In the analysis it was observed that heavy, moderate and light levels of alcohol intake were statistically related to stroke in cases and controls. The meta-analysis by Xiaoyan Zhang BA et al found a light-moderate drinking trend not statistically significant. There was evidence of an increase in the risk of stroke at the highest level relative to a heavy alcohol-drinking history. ${ }^{[13]}$

The cases and controls were further distributed in the study, depending on the duration of alcohol consumption. It was found that duration of alcohol consumption was significantly associated with occurrence of stroke. Previous study conducted by Matti $\mathrm{H}$ et al showed no link between duration of alcohol consumption and stroke but suggested that recent heavy alcohol consumption but not previous heavy drinking was an independent risk factor for stroke. ${ }^{[14]}$ 
Conclusion: The study revealed that, the odds (chance) of getting stroke among alcoholic was virtually two times more in alcoholic as compared to non-alcoholic. Male married patients who drank heavily had a higher risk of stroke than light/moderate alcoholics. Occasional alcohol consumption was less likely to get stroke compared to frequent and heavy alcohol consumption which highlights the need for a rapid cessation of alcohol consumption.

\section{Declaration:}

Funding: Nil

Conflict of Interest: Nil

\section{References:}

1. What is a Stroke? Internet Stroke Center. Available from: http://www.webcache.googleusercontent.com/search?q= cache: qimDlScia6wJ: www.strokecenter.org/patients/aboutstroke $/$ what-is-astroke $/+\& c d=3 \& h l=e n \& c t=c \operatorname{lnk} \& g l=$ in. Last Accessed 05-06-2021

2. India. National Programme for Prevention and Control of Diabetes, Cardiovascular Disease and Stroke. A Manual for Medical Officer Developed under the Government of India- WHO Collaborative. 2008. Available from: http://www.searo. who.int/india/topics/cardiovascular_diseases/NCD_Resource S_COMBINED_MANUAL_for_medical_off icer.pdf. Last Accessed 05-06-2021

3. National Center for Chronic Disease Prevention and Health Promotion, Division for Heart Disease and Stroke Prevention Type of Stroke | cdc.gov. January 31, 2020 Available from: https://www.cdc.gov/ stroke/type_of_stroke.htm. Last Accessed 05-06-2021

4. Sorganvi V, Kulkarni M S, Kadeli D, and Atherga S. Risk Factors for Stroke: A Case Control Study. IJCRR. 2014; 6 (3):46-52
5. Feroz S, Selim S, Ahammed A, Chowdhury RA, Chowdhury SH, Karim MN., \& Rahman MR. Comorbid Risk Factors for Acute Stroke: A Case-Control Study in Tertiary Care Hospital of Bangladesh. Journal of National Institute of Neurosciences Bangladesh. 2017; 2(2): 84-88. https://doi.org/10.3329/ jninb.v2i2.34100

6. Abu-Odah H, Abed Y, Abu-Hamad B. Risk Factors of Stroke in Patients Admitted inEuropean Gaza Hospital, Gaza Strip: A Case Control Study in Medical Unit Setting. J Neurol Disord StrokeVol. 2.2014.

7. National Heart, Lung, and Blood Institute (NHLBI) [Internet]. Stroke May 29-30 2019. Available from: https://www.nhlbi. nih.gov/healthtopics/stroke\#Risk-Factors.

8. O'Donnell MJ, Xavier D, Liu L,Zhang H, Chin SL, Melacini PR et al. Risk factors for ischaemicand intracerebral haemorrhagic stroke in 22 countries (the INTERSTROKE study): a case control study.JLancet2010 Jul 10;376(9735):112-23

9. AbelEL, Kruger ML, Friedl J, How do physician define "light", "moderate" and " heavy" drinking?, Alcoholism clinical and experimental research. 1998; 22(5): 979-984.

10. Zhang C, Qin Y-Y, Chen Q Jiang H, Chen X-Z, Xu C-L, et al. Alcohol intake andrisk of stroke: A dose-response meta-analysis of prospective studies. Int J Cardiol; 2014; 174(3):669-77.

11. Larsson SC, Wallin A, Wolk A, Markus HS. Differing association of alcohol consumption with different stroke types: a systematic review and meta-analysis. BMC Med. 2016; 14(1):178. 24. doi:10.1186/s12916-016-0721-4

12. Lee SJ, Cho YJ, Kim JG, Ko Y, Hong KS, Park JM, et al. Moderate alcohol intakereduces risk of ischemic stroke in Korea. Neurology 2015.85(22):1950-6.

13. Zhang, X.; Shu, L.; Si, C.; Yu, X.; Gao, W.; Liao, D.; Zhang, L.; Liu, X.; Zheng, P.Dietary Patterns and Risk of Stroke in Adults: A Systematic Review and Metaanalysisof Prospective Cohort Studies. J. Stroke Cerebrovasc Dis. 2015; 24, 2173-2182.

14. Hillbom M, Numminen H, Juvela S. Recent heavy drinking of alcohol and embolic stroke. Stroke.1999; 30(11):2307-12. 